\title{
Characterization of LhSorP5CS, a gene catalyzing proline synthesis in Oriental hybrid lily Sorbonne: molecular modelling and expression analysis
}

\author{
Le Wang ${ }^{1,2}$, Zhihong Guo 1 , Yubao Zhang ${ }^{1}$, Yajun Wang ${ }^{1}$, Guo Yang ${ }^{1}$, Liu Yang ${ }^{1,2}$, Ruoyu Wang \\ and Zhongkui Xie ${ }^{1 *}$
}

\begin{abstract}
Background: Abiotic stresses negatively affect plant growth and flower production. In plants, P5CS proteins are key enzymes that catalyzed the rate-limiting steps of proline synthesis, and proline is a well-known osmoprotectant that is closely related to abiotic stress tolerance. However, information about the P5CS genes, their effects on proline accumulation, and their role in abiotic stress tolerance in Lilium is still lacking.

Results: We isolated and characterized a novel gene (LhSorP5CS) from Oriental hybrid lily cultivar Sorbonne. Phylogenetic analysis indicated that LhSorP5CS is a member of the P5CS family. The three-dimensional structure of LhSorP5CS predicted by homology modeling showed high similarity to its correspondant human P5CS template. Further gene expression analysis revealed that LhSorP5CS expression was up-regulated by $\mathrm{NaCl}$, mannitol, and ABA, and that stressexposed plants accumulated proline at a significantly higher level than in the control.

Conclusions: LhSorP5CS characterized in this study is involved in proline synthesis in lily, and that it might play an important role in abiotic stress tolerance. However, there should be other P5CS homologues in the lily genome, and some of them could be highly stress-induced and more important for proline accumulation. Future studies on P5CS family genes would be of great importance to proline-related stress tolerance in lily.
\end{abstract}

Keywords: LhSorP5CS gene, Homology modeling, Proline, Abiotic stress, Lilium spp.

\section{Background}

Abiotic stresses such as salinity, drought, heat, and water-logging negatively affect plant growth. These environmental constraints further restrict the range of sites that are suitable for cultivation, and cause decreased agricultural productivity around the world (Zhu 2001). To protect themselves against adverse conditions, plants have evolved many physiological, cellular, and molecular mechanisms (Tan et al. 2013). One of the most well-studied protective mechanisms relates to proline metabolism.

\footnotetext{
*Correspondence: wxhcas@lzb.ac.cn

${ }^{1}$ Gaolan Station of Agricultural and Ecological Experiment, Northwest Institute of Eco-Environment and Resources, Chinese Academy of Sciences, Lanzhou 730000, China

Full list of author information is available at the end of the article
}

Stress responses in plants are often accompanied by the accumulation of proline in different tissues (Verbruggen and Hermans 2008). The accumulated proline can function as an intracellular osmolyte (Chinnusamy et al. 2005), a scavenger for reactive oxygen species (ROS) (Matysik et al. 2002), a maintainer of cell structure (Verslues et al. 2006), and a signaling molecule that primes multiple stress response pathways (Maggio et al. 2002). More recent findings have connected proline to redox status (Sharma et al. 2011; Shinde et al. 2016), and proline was considered as a storage of both energy and reducing potential (Szabados and Savoure 2010). Besides, proline metabolism was also involved in programmed cell death and plant-pathogen interaction. Accumulation of proline 
was observed at the site of infection during incompatible interaction in Arabidopsis (Fabro et al. 2004).

The metabolic pathways for proline synthesis have been deciphered and proven to be evolutionarily conserved from bacteria to higher organisms (Rai and Penna 2013). In plants, two routes for proline synthesis are known to exist, a glutamate pathway and an ornithine pathway (Adams and Frank 1980; Delauney and Verma 1993). However, the glutamate-derived pathway is the major route for proline synthesis under stress conditions (Trovato et al. 2008). In this pathway, glutamate is reduced by $\Delta 1$-pyrroline-5-carboxylate synthetase (P5CS) to form $\gamma$-glutamate-semialdehyde (GSA) in a manner that depends on ATP and NADPH, then GSA cyclized itself to form P5C (Liang et al. 2013). These reactions are ratelimiting steps in proline synthesis and are catalyzed by P5CS; thus, P5CS genes play an important role in stress tolerance.

Given that P5CS plays such an important role in proline synthesis, many attempts to improve stress tolerance by increasing proline production have centered on P5CS genes. For example, overexpression of P5CSF129A resulted in increased proline production and enhanced salt tolerance in indica rice (Oryza sativa L.) (Kumar et al. 2010). In citrus species, plants overproduced proline exhibited superior osmotic adjustment and higher photosynthetic rates under drought stress (Molinari et al. 2004).

In the present study, we cloned the full-length cDNA sequence of a P5CS gene (LhSorP5CS) from the Oriental hybrid lily cv. Sorbonne (Lilium spp.) by rapid amplification of cDNA ends (RACE). Phylogenetic analysis of the deduced LhSorP5CS amino acid sequence by comparison with other P5CS proteins in plants indicated that LhSorP5CS was a member of the P5CS family. We investigated the expression of LhSorP5CS in different tissues and in response to salt, drought, and abscisic acid (ABA) stresses by means of real-time quantitative polymerase chain reaction (qPCR). To our knowledge, LhSorP5CS is the first P5CS gene that has been functionally characterized in Lilium.

\section{Methods}

\section{Plant growth conditions}

The Oriental hybrid lily cultivar Sorbonne was planted in the greenhouse of the Gaolan Agricultural and Ecological Experiment Station, in northern China. Bulbs 10-12 cm in diameter were grown in plastic pots containing peat and kept under a $16 \mathrm{~h}$ light $/ 8 \mathrm{~h}$ dark photoperiod at $22^{\circ} \mathrm{C}$.

\section{Total RNA extraction and first-strand CDNA synthesis}

Total RNA was extracted with RNAprep Pure Plant kits (TIANGEN Corporation, Beijing, China) following the manufacturer's instructions. We used $1 \mu \mathrm{g}$ of total RNA for first-strand cDNA synthesis. First-strand cDNA was synthesized using the PrimeScript first-strand cDNA synthesis kit (Takara, Dalian, China) or the SMARTer RACE cDNA Amplification Kit (for RACE cloning; Clontech, USA). Synthesized cDNA was subsequently diluted to a final concentration of $20 \mathrm{ng} \mu \mathrm{L}^{-1}$ with nuclease-free water for use in cloning.

\section{Full length cloning of LhSorP5CS}

We designed a degenerate primer set (P5CS-Deg-F: 5'-GGNATHTTYTGGGAYAAYGA-3'; P5CS-Deg-R: $5^{\prime}$-GTYTCCATNGCRTTRCANGC-3') based on conserved regions for plant P5CS enzymes. PCR was performed in a $25-\mu \mathrm{L}$ reaction mixture containing $20 \mathrm{ng}$ of template cDNA, $200 \mu \mathrm{mol} \mathrm{L}^{-1}$ of dNTPs, $1.0 \mu \mathrm{mol} \mathrm{L}^{-1}$ of each primer, and $1.25 \mathrm{U}$ of TransTaq HiFi DNA polymerase (TransGen, Beijing, China). PCR products were gel-purified, ligated into the pMD-18T vector (Takara, Dalian, China), and sequenced (Sangon, Shanghai, China). Based on the partial coding sequence that we obtained, we designed gene-specific primers for 5'-RACE (5RACE-GSP-1: 5'-GGTCGGCCTTCAACTC CATCGCC-3'; 5RACE-GSP-2: 5'-AGCCCCGCTAA GCTGTCATTGTCCC-3') and $3^{\prime}$-RACE (3RACE-GSP-1: 5'-GATCTCGTGATTCCGAGGGGTAGC-3'; 3RACEGSP-2: $5{ }^{\prime}$-CGCCAAGACAGATTACCCAGCAGC-3'). The full-length sequence was obtained by assembling the 5 -RACE sequence, the partial coding sequence, and the $3^{\prime}$-RACE sequence.

\section{In silico analysis of $\operatorname{LhSorP5CS}$}

The open reading frame (ORF) of the full-length cDNA of the LhSorP5CS sequence was predicted using the ORF finder. Homology of the deduced P5CS protein with other members of this family was verified by querying the NCBI database using the BLAST software. Multiple alignment of sequences was performed using the Clustal W program. A phylogenetic tree of the P5CS proteins was constructed using the Mega 4.1 software (Tamura et al. 2007) using the neighbor-joining method.

\section{Three-dimensional structure prediction by homology modeling}

Prediction of 3D structure was carried out via accessing Swiss-Model(Schwede et al. 2003), a web-based protein structure prediction tool. Superposition of the built LhSorP5CS model on the template was achieved by Swiss PDB viewer programme. The root mean square deviation (RMSD),which measure the average distance between the backbone atoms of superposed proteins, was calculated using the PDBeFold programme (Krissinel 2007). Evaluation of stereochemical quality of the LhSorP5CS model 
was carried out by using Procheck V.3.5.4 (Laskowski et al. 1993).

\section{Expression analysis of LhSorP5CS in different tissues and under various stress treatments}

Tissues (root, stem, leaf, petal, and scale) were sampled from 2-month-old fully bloomed plants and used as the materials for tissue-specific expression analysis. For exogenous stress treatments, the bulbs of 30-day-old seedlings were drenched with $500 \mathrm{~mL}$ solutions containing $500 \mathrm{mM}$ mannitol, $500 \mathrm{mM} \mathrm{NaCl}$, or $2 \mu \mathrm{M} \mathrm{ABA}$. Leaf samples were taken $0,2,4,8,12$, and $24 \mathrm{~h}$ after the stress treatments. All samples were frozen immediately in liquid nitrogen and kept in a refrigerator at $-80{ }^{\circ} \mathrm{C}$ until use.

After RNA extraction, first strand cDNA synthesis of templates for qPCR was carried out using the HiScript II Q RT SuperMix for qPCR kits (Vazyme Biotech, Nanjing, China), and contamination with genomic DNA was removed by using the gDNA wiper following the manufacturer's protocol. Real-time qPCR was performed using an MX3000P qPCR thermocycler (Stratagene, USA). The Oriental hybrid lily cv. Sorbonne polyubiquitin4 gene (GenBank accession no. DW718023) was used as the reference gene (Yamagishi 2011). The P5CS-specific primer set (P5CS-q-F: 5'-GCTTTCATCAGGGACGCCAAG-3'; P5CS-q-R: $\quad 5^{\prime}$-CAACACCAACCGCACCAGAAG-3') was designed using OligoArchitect (Fourie et al. 2014), online software developed by Sigma-Aldrich. qPCR was performed using the AceQ qPCR SYBR Green Master Mix kit (Vazyme Biotech, Nanjing, China) according to the manufacturer's instructions. The following protocol was used for amplification: pre-denaturation for $10 \mathrm{~min}$ at $95{ }^{\circ} \mathrm{C}$, followed by 40 cycles of $94^{\circ} \mathrm{C}$ for $15 \mathrm{~s}$ and $60^{\circ} \mathrm{C}$ for $30 \mathrm{~s}$. Relative changes in gene expression were analyzed by using the $2^{-\Delta \Delta C T}$ method, and the $C_{T}$ values used were from three independent biological replicates.

\section{Proline content determination}

We determined the free proline content according to the method proposed by Bates et al. (1973). In summary, $0.5 \mathrm{~g}$ fresh weight (FW) of leaf tissue was homogenized in $3 \%$ sulfosalicylic acid solution using a mortar and pestle. The homogenate was then filtered through Whatman 42 filter paper. We then added $2 \mathrm{~mL}$ of $2.5 \%$ acidic ninhydrin and $2 \mathrm{~mL}$ of acetic acid to $2 \mathrm{~mL}$ of the filtrate. After boiling for $1 \mathrm{~h}$, the mixture was placed on ice and proline was extracted using $4 \mathrm{~mL}$ of toluene. The proline content per unit FW was calculated based on the absorbance readings at $520 \mathrm{~nm}$ using a UV-1200 spectrometer (Macy, Shanghai, China). Pure proline was used as the analytical standard.

\section{Statistical analysis}

All experiments were repeated three times, with three replicates each time. We used version 8 of the SAS software for statistical analysis and the Origin Pro 8.1 software to plot the data.

\section{Results}

Molecular cloning and sequence analysis of $L h$ SorP5CS

The cloned full-length LhSorP5CS cDNA contained $2645 \mathrm{bp}$, comprising a $5^{\prime}$ untranslated region (UTR) of $170 \mathrm{bp}$, a predicted ORF of $2139 \mathrm{bp}$, and a $3^{\prime}$ UTR of $196 \mathrm{bp}$. The ORF encoded a protein of 712 amino acids with a theoretical molecular mass of $77.3 \mathrm{kDa}$ and a predicted isoelectric point (pI) of 5.71. The LhSorP5CS cDNA sequence has been submitted to Genbank and deposited with the accession number KU057356.

Multiple alignment showed that the deduced LhSorP5CS amino acid sequence exhibited high homology with other P5CS proteins from plants, such as MaAAAP5CS (83\%), TaP5CS (77\%), OsP5CS1 (79\%), and ZmP5CS (79\%). The LhSorP5CS protein sequence was compared with homologues from Oryza sativa and Arabidopsis thaliana (Additional file 1: Figure S1), and conserved regions included the ATP binding site, two Leu-rich domains, the Glu-5-kinase domain, the $\mathrm{NAD}(\mathrm{P}) \mathrm{H}$ binding domain, and the GSA-DH domain. A conserved phenylalanine residue (Phe, at position 125), which functioned in proline feedback inhibition, was also found in LhSorP5CS.

A phylogenetic tree was built to analyze the evolutionary relationship of the LhSorP5CS protein with P5CS proteins from other species. Phylogenetic analysis revealed that the P5CS proteins in plants clustered into two major groups: the monocot group and the dicot group. LhSorP5CS was most closely related to MaAAAP5CS in the monocot group (Fig. 1). These findings suggest that the LhSorP5CS that we cloned is a new gene in the P5CS family, and that the putative LhSorP5CS might be involved in proline synthesis in lily.

\section{Predicted three-dimensional structure of the LhSorP5CS protein}

The deduced LhSorP5CS protein sequence was submitted to server to BLAST against the Swiss-Model template library to obtain the best template. The searching result revealed that human P5CS (2h5g.1.A) which share $50.12 \%$ identity with LhSorP5CS was the best alignment. Thus, the human P5CS protein was used as template (Fig. 2a) to build the LhSorP5CS 3D model (Fig. 2b). Superposition (Fig. 2c) of the built LhSorP5CS model with the human P5CS template showed that 3D structure of these two proteins were highly similar, and the 


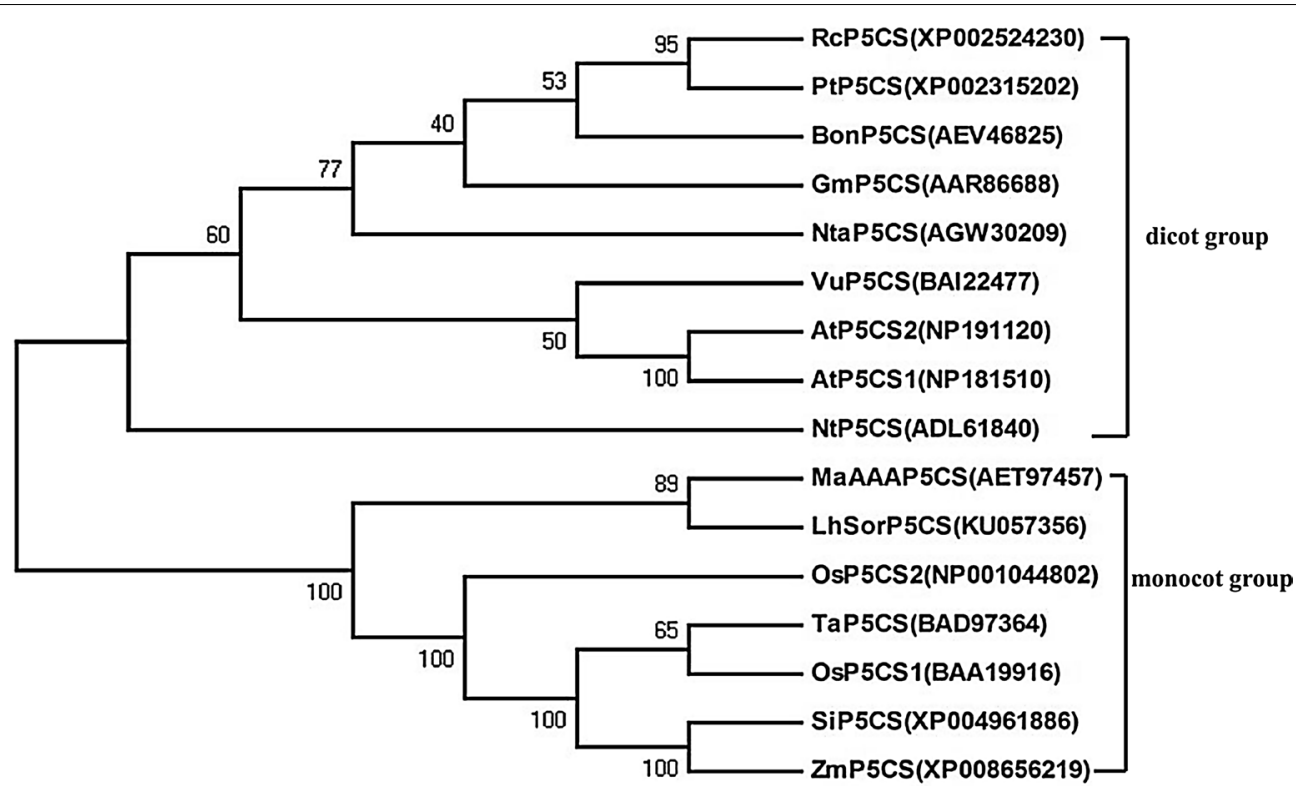

Fig. 1 Phylogram of LhSorP5CS and other P5CSs from different species. Phylogenetic relationship of LhSorP5CS with AtP5CS1 (Arabidopsis thaliana P5CS1), AtP5CS2 (A. thaliana P5CS2), OsP5CS1 (Oryza sativa P5CS1), OsP5CS2 (O. sativa P5CS2), RcP5CS (Ricinus communis P5CS), PtP5CS (Populus trichocarpa P5CS), BonP5CS (Boehmeria nivea P5CS), GmP5CS (Glycine max P5CS), NtaP5CS (Nitraria tangutorum P5CS), VuP5CS (Vigna unguiculata P5CS), NtP5CS (Nicotiana tabacum P5CS), MaAAAP5CS (Musa acuminata AAA Group P5CS), TaP5CS (Triticum aestivum P5CS), SiP5CS (Setaria italica P5CS), and ZmP5CS (Zea mays P5CS). Numbers in brackets refer to the sequences accession number in GenBank

calculated RMSD of the superposed proteins was 0.45 . Ramachandran plot (Additional file 2: Figure S2) showed that $90.3 \%$ of residues in the LhSorP5CS model were in the most favored regions, $8.7 \%$ in the additional allowed regions, $0.5 \%$ in the generously allowed regions; while only $0.5 \%$ of residues were in the disallowed regions. The result that over $90 \%$ of the residues were in the most favored regions indicated that the LhSorPR5 model presented was well built.

\section{Expression analysis}

\section{Tissue-specific expression of LhSorP5CS}

Figure 3 shows that LhSorP5CS expression was detected in all five tissues (root, stem, leaf, petal, and scale), but the abundance of transcripts was significantly higher in the root and stem tissues than in the other tissues. The levels of LhSorP5CS transcripts in root and stem tissues were 5.08 and 5.03 times, respectively, that in the petal tissue, which had the lowest expression. The abundance of LhSorP5CS transcripts in petal tissue was also significantly lower than that in leaf and scale tissues (which were 2.77 and 2.41 times the petal level, respectively).

\section{Response of LhSorP5CS expression to abiotic stresses}

We used the levels of LhSorP5CS transcripts to investigate whether LhSorP5CS expression was induced by salt, drought, or ABA stress. Figure 4a shows that LhSorP5CS expression was strongly and significantly induced by $\mathrm{NaCl} 2 \mathrm{~h}$ after treatment, reaching a level 6.92 times that in the control. Similarly, significant up-regulation of LhSorP5CS also occurred $2 \mathrm{~h}$ after mannitol treatment, to 1.88 times the control level (Fig. 4b); that is, the response was weaker than that to the $\mathrm{NaCl}$ treatment. Under ABA treatment, LhSorP5CS expression was significantly up-regulated by $2 \mathrm{~h}$ after treatment, reaching 1.77 times the control level, and then increased to 2.66 times the control level by $4 \mathrm{~h}$ after treatment (Fig. 4c); this was also weaker than the $\mathrm{NaCl}$ response. The rapid induction of LhSorP5CS in the presence of salt, drought, and ABA stress suggests that LhSorP5CS is an abiotic stressresponse gene in lily.

\section{Proline accumulation}

We also measured proline accumulation in leaves $12 \mathrm{~h}$ after the abiotic stress treatments, using the same samples used for qPCR. The $\mathrm{NaCl}$, mannitol, and ABA treatments all significantly increased proline accumulation in the leaves compared with the control, with values reaching $3.39,1.81$, and 1.34 times the control value, respectively (Fig. 5). These results suggest that proline accumulation was significantly induced by salt, drought, and $\mathrm{ABA}$ stress treatments in lily and that the rapid increase in LhSorP5CS expression may be involved in proline production as part of lily's stress response. 

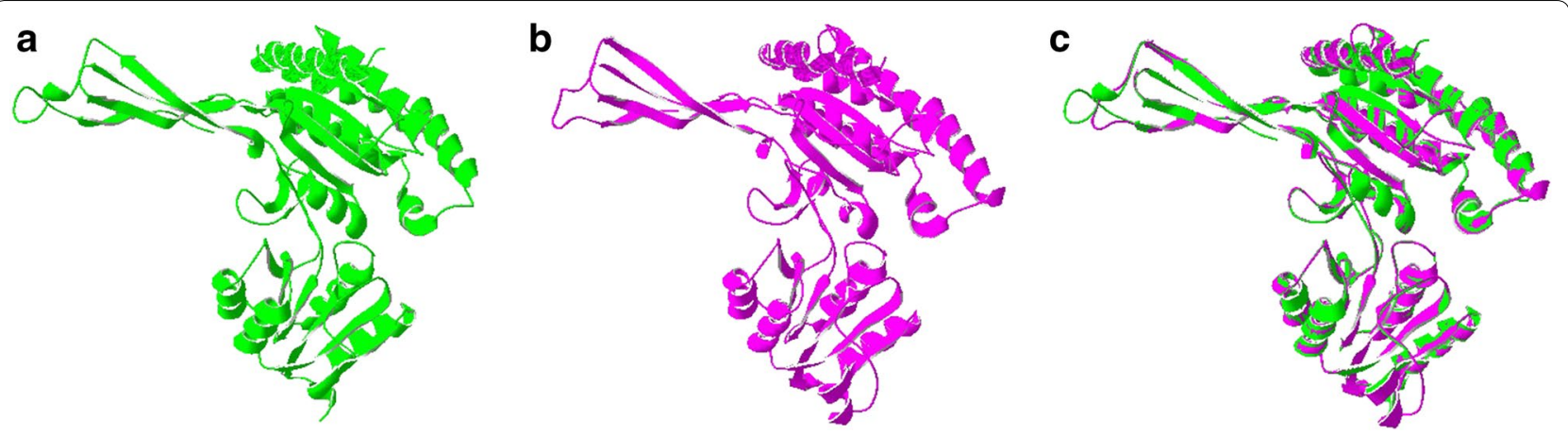

Fig. 2 Three dimensional structure of LhSorP5CS generated by homology modeling with the human P5CS as template. a Structure of the human P5CS used as template. b Predicted structure of LhSorP5CS. c Superposition of LhSorP5CS and the human P5CS protein shows highly similar structure between the two homologs

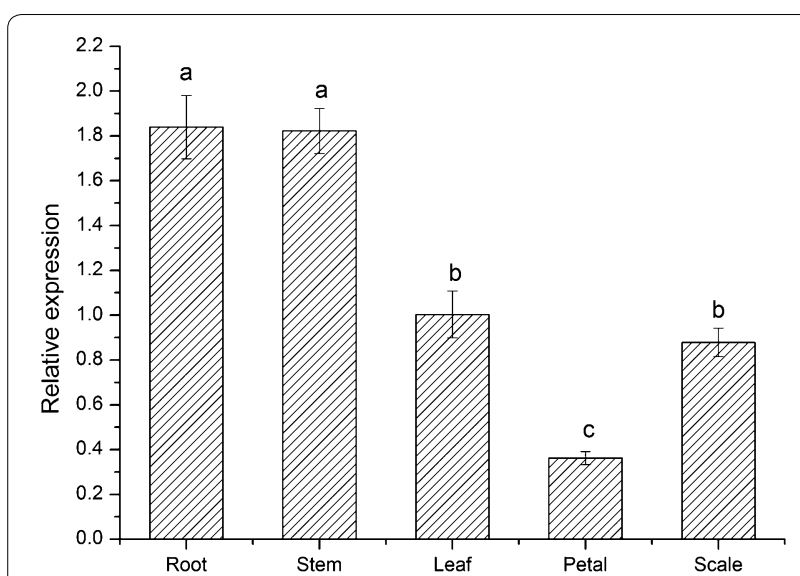

Fig. 3 Tissue-specific expression of LhSorP5CS from Oriental hybrid lily cv. Sorbonne (Lilium spp.). Transcript levels of the target gene were normalized against the polyubiquitin4 internal control. Values are mean \pm standard deviation (SD) for three replicates. Bars labeled with different letters differ significantly $(P<0.05, t$ test)

\section{Discussion}

Proline, an amino acid known to function as an osmoprotectant, a ROS scavenger, a cell structure maintainer, and a signaling molecule, plays an active role in plant stress responses. In plants, the rate-limiting steps in proline synthesis are catalyzed by P5CS. The function of the first plant P5CS gene from Vigna aconitifolia was revealed by its complementation of the pro $A$ and proB mutations in E. coli (Hu et al. 1992). Since then, P5CS homologues have been cloned from A. thaliana (Strizhov et al. 1997), O. sativa (Igarashi et al. 1997), M. truncatula (Ginzberg et al. 1998) and other species (Chen et al. 2010; Fujita et al. 1998; Zhu et al. 2012). In this study, we cloned the full-length cDNA sequence of the LhSorP5CS gene, the first P5CS gene reported in Lilium. Phylogenetic analysis revealed that LhSorP5CS was closely related to P5CS proteins from monocot plants, and had $83 \%$ identity with MaAAAP5CS. The LhSorP5CS transcript level was significantly up-regulated by salt, drought, and ABA stress.
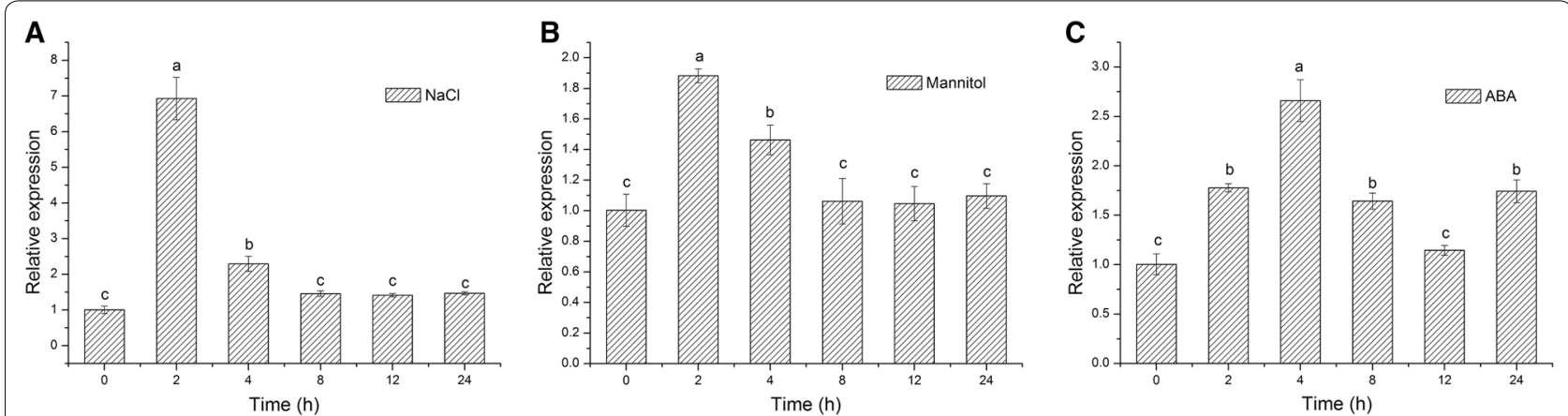

Fig. 4 LhSorP5CS expression pattern in response to abiotic stresses. LhSorP5CS transcript level was quantified in leaves of the Oriental hybrid lily Cv. Sorbonne (Lilium spp.) under the a $\mathrm{NaCl}$, b mannitol, and $\mathbf{c} \mathrm{ABA}$ treatments. Values were determined by real-time qPCR, and normalized using transcript levels of LhSorP5CS in the leaves of unstressed plants (the control), which were set to a value of 1 . Values represent the mean \pm standard deviation (SD) for three replicates. Bars labeled with different letters differ significantly $(P<0.05, t$ test). Note that the $y$-axis scale differs greatly between graphs 


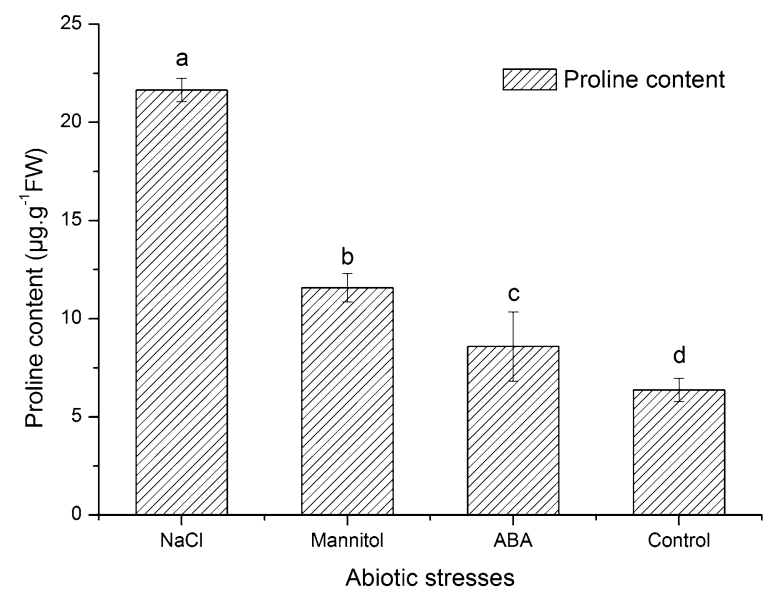

Fig. 5 Proline contents in leaves of Oriental hybrid lily cv. Sorbonne (Lilium spp.) sampled $12 \mathrm{~h}$ after treating lily plants with different abiotic stresses. Values represent mean \pm standard deviation (SD) for three replicates. Bars labeled with different letters differ significantly $(P<0.05, t$ test $)$

By $12 \mathrm{~h}$ after stress treatments, a significantly higher proline content was detected in leaf tissue, revealing proline accumulation induced by salt and drought stress in lily. This agrees with previous research that showed the accumulation of P5CS transcripts and proline in many different plant species under stress. In sweet sorghum (Sorghum bicolor) (Su et al. 2011), induction of P5CS expression was observed before proline accumulation under salt and drought stress. Similarly, rapid up-regulation of PvP5CS was observed prior to proline accumulation in Phaseolus vulgaris (Chen et al. 2009). Hence, it appears that LhSorP5CS is involved in proline synthesis and that the accumulation of proline might result from up-regulation of $L h S o r P 5 C S$ expression in lily.

Because P5CS genes appear to be the most important genes involved in catalyzing proline synthesis, they have been selected as candidate genes to improve abiotic stress tolerance by elevating in vivo proline levels. Increased proline production and enhanced tolerance to multiple stresses have been reported in several transgenic plants, including potato (Solanum tuberosum) (Hmida-Sayari et al. 2005), Arabidopsis (Chen et al. 2013), chickpea (Cicer arietinum) (Ghanti et al. 2011), and wheat (Triticum aestivum) (Vendruscolo et al. 2007). The LhSorP5CS gene that we cloned in the present study is therefore also a candidate gene for future transgenic research in lily.
In higher plants, stress-induced proline accumulation has been observed in many species, and halophytes generally accumulated more proline than glycophytes under adverse conditions (Verbruggen and Hermans 2008). Therefore, the proline level was often correlated with stress tolerance, especially for salt and drought tolerance (Chakraborty et al. 2012; Chauhan et al. 1980; Putnik-Delic et al. 2013). However, this correlation was not always consistent. A recent study of rice seedlings suggested that the proline accumulation level was not a reliable parameter for assessing tolerance of saline alkaline conditions (Lv et al. 2015). Similarly, a study by Deng (2013) suggested that there was no correlation between proline accumulation and drought tolerance in ten genotypes of Tibetan hull-less barley (Hordeum vulgare var. nudum). Hence, it is worth noting that even though proline plays a multifaceted role in plant stress adaption, its accumulation level may not be significantly associated with stress tolerance and its role may differ among species. It will be necessary to confirm this association in future research on other lily accessions.

The non-consistent correlation between proline level and stress tolerance can be ascribed to the fact that proline turnover, rather that just proline accumulation, was more important to plant growth maintaining under stress. Although many early attempts have proven that improvement of stress tolerance through elevating proline level was feasible, recent evidence (Bhaskara 2015) showed that mutation of proline dehydrogenase 1 (PDH1), the key enzyme catalyze proline catabolism, resulted in similar reduction in growth as the $p 5 c s 1$ mutant in spite of elevated proline production. Such findings emphasize the importance of proline catabolism to plant growth maintaining under stress, and underlie that the flux and turnover of proline, rather than just proline accumulation, might be more important to stress adaption for plants.

\section{Conclusions}

LhSorP5CS, the first P5CS gene cloned from Lilium, encoded a protein with 712 amino acids and a pI of 5.71 . Expression of LhSorP5CS was significantly up-regulated when the plants were exposed to salt, drought, and ABA stresses. Proline accumulation increased significantly $12 \mathrm{~h}$ after the stress treatments. These findings suggest that LhSorP5CS plays an important role in proline synthesis and stress tolerance in lily. However, there should be other P5CS homologues in the lily genome, and some 
of them could be highly stress-induced and more important for proline accumulation. Future studies on P5CS family genes would be of great importance to prolinerelated stress tolerance in lily.

\section{Additional files}

Additional file 1: Figure S1. Multiple sequence alignment of LhSorP5CS with P5CSs from other plants. Alignment of the putative amino acid sequence of the Oriental hybrid lily cv. Sorbonne LhSorP5CS with sequences of Arabidopsis thaliana AtP5CS1 (Genbank accession no. NP181510) and AtP5CS2 (Genbank accession no. NP191120), and Oryza sativa OsP5CS1 (Genbank accession no. BAA19916) and OsP5CS2 (Genbank accession no. NP001044802). Identical residues are shaded in dark blue, highly similar residues are shaded in pink and similar residues are shaded in light blue. Upperlined sequences represent putative ATP and NAD(P)H-binding sites, conserved Glu-5-kinase and GSA-DH domains, and leucine (Leu)-rich regions. The conserved phenylalanine (Phe) residue that functions in proline feedback inhibition is indicated by an asterisk (*).

Additional file 2: Figure S2. Ramachandran plot of the built model of LhSorP5CS. The red, yellow, light yellow and white regions represent the most favored, additional allowed, generously allowed, and disallowed regions respectively.

\section{Abbreviations}

P5CS: $\triangle 1$-pyrroline-5-carboxylate synthetases; GSA: $\gamma$-glutamatesemialdehyde; RACE: rapid amplification of CDNA ends; ABA: abscisic acid; ORF: open reading frame; UTR: untranslated region.

\section{Authors' contributions}

ZX and YW conceived and designed the experiments; LW and ZG performed the experiments; $Y Z$ and $L Y$ analyzed the data; LW, GY and RW wrote the manuscript. All authors read and approved the final manuscript.

\section{Author details \\ ${ }^{1}$ Gaolan Station of Agricultural and Ecological Experiment, Northwest Institute of Eco-Environment and Resources, Chinese Academy of Sciences, Lanzhou 730000, China. ${ }^{2}$ University of Chinese Academy of Sciences, Bei- jing 100049, China.}

\section{Acknowledgements}

This research was supported by the Ningxia Agricultural Comprehensive Development Office (NTKJ2015-05-01), the National Natural Science Foundation of China (Grant No. 31201651) and the "100 Talents Programme" of the Chinese Academy of Sciences (No. 27Y127L41002).

\section{Competing interests}

The authors declare that they have no competing interests.

Received: 30 September 2016 Accepted: 7 January 2017

Published online: 18 January 2017

\section{References}

Adams E, Frank L (1980) Metabolism of proline and the hydroxyprolines. Annu Rev Biochem 49:1005-1061

Bates LS, Waldren RP, Teare ID (1973) Rapid determination of free proline for water-stress studies. Plant Soil 39:205-207

Bhaskara GB, Yang TH, Verslues PE (2015) Dynamic proline metabolism: importance and regulation in water limited environments. Front Plant Sci 6:484

Chakraborty K, Sairam RK, Bhattacharya RC (2012) Salinity-induced expression of pyrrolline-5-carboxylate synthetase determine salinity tolerance in Brassica spp. Acta Physiol Plant 34:1935-1941

Chauhan RPS, Chauhan CPS, Kumar D (1980) Free proline accumulation in cereals in relation to salt tolerance. Plant Soil 57:167-175
Chen JB, Wang SM, Jing RL, Mao XG (2009) Cloning the PvP5CS gene from common bean (Phaseolus vulgaris) and its expression patterns under abiotic stresses. J Plant Physiol 166:12-19

Chen JB, Zhang XY, Jing RL, Blair MW, Mao XG, Wang SM (2010) Cloning and genetic diversity analysis of a new P5CS gene from common bean (Phaseolus vulgaris L.). Theor Appl Genet 120:1393-1404

Chen JB, Yang JW, Zhang ZY, Feng XF, Wang SM (2013) Two P5CS genes from common bean exhibiting different tolerance to salt stress in transgenic Arabidopsis. J Genet 92:461-469

Chinnusamy V, Jagendorf A, Zhu JK (2005) Understanding and improving salt tolerance in plants. Crop Sci 45:437-448

Delauney AJ, Verma DPS (1993) Proline biosynthesis and osmoregulation in plants. Plant J 4:215-223

Deng G, Liang J, Xu D, Long H, Pan Z, Yu M (2013) The relationship between proline content, the expression level of P5CS (delta(1)-pyrroline-5-carboxylate synthetase), and drought tolerance in Tibetan hulless barley (Hordeum vulgare var. nudum). Russ J Plant Physiol 60:693-700

Fabro G, Kovacs I, Pavet V, Szabados L, Alvarez ME (2004) Proline accumulation and AtP5CS2 gene activation are induced by plant-pathogen incompatible interactions in Arabidopsis. Mol Plant Microbe Interact 17:343-350

Fourie JJ, Joubert A, Labuschagne M, Beugnet F (2014) New method using quantitative PCR to follow the tick blood meal and to assess the antifeeding effect of topical acaricide against Rhipicephalus sanguineus on dogs. Comp Immunol Microbiol Infect Dis 37:181-187

Fujita T, Maggio A, Garcia-Rios M, Bressan RA, Csonka LN (1998) Comparative analysis of the regulation of expression and structures of two evolutionarily divergent genes for delta(1)-pyrroline-5-carboxylate synthetase from tomato. Plant Physiol 118:661-674

Ghanti SKK, Sujata KG, Kumar BMV, Karba NN, Reddy KJ, Rao MS, Kishor PBK (2011) Heterologous expression of P5CS gene in chickpea enhances salt tolerance without affecting yield. Biol Plant 55:634-640

Ginzberg I, Stein H, Kapulnik Y, Szabados L, Strizhov N, Schell J, Koncz C, Zilberstein A (1998) Isolation and characterization of two different CDNAs of delta(1)-pyrroline-5-carboxylate synthase in alfalfa, transcriptionally induced upon salt stress. Plant Mol Biol 38:755-764

Hmida-Sayari A, Gargouri-Bouzid R, Bidani A, Jaoua L, Savoure A, Jaoua S (2005) Overexpression of delta(1)-pyrroline-5-carboxylate synthetase increases proline production and confers salt tolerance in transgenic potato plants. Plant Sci 169:746-752

Hu CAA, Delauney AJ, Verma DPS (1992) A bifunctional enzyme (delta1-pyrroline-5-carboxylate synthetase) catalyzes the 1st 2 steps in proline biosynthesis in plants. Proc Natl Acad Sci USA 89:9354-9358

Igarashi Y, Yoshiba Y, Sanada Y, YamaguchiShinozaki K, Wada K, Shinozaki K (1997) Characterization of the gene for delta(1)-pyrroline-5-carboxylate synthetase and correlation between the expression of the gene and salt tolerance in Oryza sativa L. Plant Mol Biol 33:857-865

Krissinel E (2007) On the relationship between sequence and structure similarities in proteomics. Bioinformatics 23:717-723

Kumar V, Shriram V, Kishor PBK, Jawali N, Shitole MG (2010) Enhanced proline accumulation and salt stress tolerance of transgenic indica rice by overexpressing P5CSF129A gene. Plant Biotechnol Rep 4:37-48

Laskowski RA, Macarthur MW, Moss DS, Thornton JM (1993) Procheck-a program to check the stereochemical quality of protein structures. J Appl Crystallogr 26:283-291

Liang XW, Zhang L, Natarajan SK, Becker DF (2013) Proline mechanisms of stress survival. Antioxid Redox Signal 19:998-1011

Lv BS, Ma HY, Li XW, Wei LX, Lv HY, Yang HY, Jiang CJ, Liang ZW (2015) Proline accumulation is not correlated with saline-alkaline stress tolerance in rice seedlings. Agron J 107:51-60

Maggio A, Miyazaki S, Veronese P, Fujita T, Ibeas Jl, Damsz B, Narasimhan ML, Hasegawa PM, Joly RJ, Bressan RA (2002) Does proline accumulation play an active role in stress-induced growth reduction? Plant J 31:699-712

Matysik J, Alia B Bhalu, Mohanty P (2002) Molecular mechanisms of quenching of reactive oxygen species by proline under stress in plants. Curr Sci India 82:525-532

Molinari HBC, Marur CJ, Bespalhok JC, Kobayashi AK, Pileggi M, Leite RP, Pereira LFP, Vieira LGE (2004) Osmotic adjustment in transgenic citrus rootstock Carrizo citrange (Citrus sinensis Osb. $\times$ Poncirus trifoliata L. Raf.) overproducing proline. Plant Sci 167:1375-1381

Putnik-Delic M, Maksimovic I, Venezia A, Nagl N (2013) Free proline accumulation in young sugar beet plants and in tissue culture explants under 
water deficiency as tools for assessment of drought tolerance. Rom Agric Res 30:141-148

Rai AN, Penna S (2013) Molecular evolution of plant P5CS gene involved in proline biosynthesis. Mol Biol Rep 40:6429-6435

Schwede T, Kopp J, Guex N, Peitsch MC (2003) SWISS-MODEL: an automated protein homology-modeling server. Nucleic Acids Res 31:3381-3385

Sharma S, Villamor JG, Verslues PE (2011) Essential role of tissue-specific proline synthesis and catabolism in growth and redox balance at low water potential. Plant Physiol 157:292-304

Shinde S, Villamor JG, Lin W, Sharma S, Verslues PE (2016) Proline coordination with fatty acid synthesis and redox metabolism of chloroplast and mitochondria. Plant Physiol 172:1074-1088

Strizhov N, Abraham E, Okresz L, Blickling S, Zilberstein A, Schell J, Koncz C, Szabados L (1997) Differential expression of two P5CS genes controlling proline accumulation during salt-stress requires $A B A$ and is regulated by ABA1, ABI1 and AXR2 in Arabidopsis. Plant J 12:557-569

Su M, Li XF, Ma XY, Peng XJ, Zhao AG, Cheng LQ, Chen SY, Liu GS (2011) Cloning two P5CS genes from bioenergy sorghum and their expression profiles under abiotic stresses and MeJA treatment. Plant Sci 181:652-659

Szabados L, Savoure A (2010) Proline: a multifunctional amino acid. Trends Plant Sci 15:89-97

Tamura K, Dudley J, Nei M, Kumar S (2007) MEGA4: molecular evolutionary genetics analysis (MEGA) software version 4.0. Mol Biol Evol 24:1596-1599
Tan CM, Chen RJ, Zhang JH, Gao XL, Li LH, Wang PR, Deng XJ, Xu ZJ (2013) OsPOP5, a prolyl oligopeptidase family gene from rice confers abiotic stress tolerance in Escherichia coli. Int J Mol Sci 14:20204-20219

Trovato M, Mattioli R, Costantino P (2008) Multiple roles of proline in plant stress tolerance and development. Rend Lincei 19:325-346

Vendruscolo ECG, Schuster I, Pileggi M, Scapim CA, Correa Molinari HB, Marur CJ, Esteves Vieira LG (2007) Stress-induced synthesis of proline confers tolerance to water deficit in transgenic wheat. J Plant Physiol 164:1367-1376

Verbruggen N, Hermans C (2008) Proline accumulation in plants: a review. Amino Acids 35:753-759

Verslues PE, Agarwal M, Katiyar-Agarwal S, Zhu J, Zhu JK (2006) Methods and concepts in quantifying resistance to drought, salt and freezing, abiotic stresses that affect plant water status. Plant J 45:523-539

Yamagishi M (2011) Oriental hybrid lily Sorbonne homologue of LhMYB12 regulates anthocyanin biosyntheses in flower tepals and tepal spots. Mol Breed 28:381-389

Zhu JK (2001) Plant salt tolerance. Trends Plant Sci 6:66-71

Zhu XY, Li XP, Zou Y, Chen WX, Lu WJ (2012) Cloning, characterization and expression analysis of delta(1)-pyrroline-5-carboxylate synthetase (P5CS) gene in harvested papaya (Carica papaya) fruit under temperature stress. Food Res Int 49:272-279

\section{Submit your manuscript to a SpringerOpen ${ }^{\circ}$ journal and benefit from:}

- Convenient online submission

- Rigorous peer review

- Immediate publication on acceptance

- Open access: articles freely available online

- High visibility within the field

- Retaining the copyright to your article 